\title{
REVIEW
}

\section{Improving the utility of prostate specific antigen (PSA) in the diagnosis of prostate cancer: the use of PSA derivatives and novel markers}

\section{S Jain, A G Bhojwani, J K Mellon}

Postgrad Med J 2002;78:646-650

Prostate specific antigen (PSA) testing is now a routine part of the investigation of men with suspected prostate cancer. While a very useful test it still has its problems, in particular its lack of specificity means abnormal results are often caused by benign disease. This review describes the current problems with PSA testing in prostate cancer diagnosis and highlights potential ways in which these may be reduced.

See end of article for authors' affiliations

....................

Correspondence to: Mr Sunjay Jain, Division of Urology, University of Leicester, Leicester General Hospital, Gwendolen Road, Leicester, LE5 4PW, UK; sj34@le.ac.uk

Submitted 28 May 2002 Accepted 22 July 2002
$\mathrm{P}$ rostate specific antigen (PSA) is now a well established tumour marker that aids the diagnosis, staging, and follow up of prostate cancer. Discovered in the early 1970s, the first commercial assay for PSA was approved in 1986 and the first clinical applications were reported soon after, showing it to be superior to the previously used marker, prostatic acid phosphatase.

Biochemically, PSA is a serine protease, also known as human kallikrein 3 (hK3). It is a member of a homologous group of proteases, the human glandular kallikrein family that are coded for by genes located on chromosome 19q. ${ }^{2}$ Its physiological function is to dissolve the gel formed after ejaculation and thereby permit sperm movement in the female genital tract. ${ }^{3}$

The majority of PSA produced by the prostate is excreted in the semen but a small proportion "leaks" into the systemic circulation. Studies by Stamey et al showed that, on a weight for weight basis, prostate cancer tissue released 30 times more PSA into the circulation than normal prostate tissue, perhaps because of loss of normal tissue architecture. ${ }^{1}$ Unfortunately other diseases of the prostate such as benign prostatic hyperplasia $(\mathrm{BPH})$ and chronic inflammation also cause increased PSA release into the circulation. As essentially the same group of men are at risk of $\mathrm{BPH}$, chronic infection and cancer this has somewhat reduced the utility of serum PSA as a diagnostic test.

Figure 1 illustrates this problem. The traditional cut off of $4 \mathrm{ng} / \mathrm{ml}$ that has been used to

Table 1 The likelihood of having prostate cancer at different PSA values

\begin{tabular}{ll}
\hline PSA $(\mathrm{ng} / \mathrm{ml})$ & Men with prostate cancer $(\%)$ \\
\hline $4-10$ & $22-27$ \\
$>10$ & $50-67$ \\
\hline
\end{tabular}

identify which men need further investigation has a sensitivity for detecting cancer of approximately $75 \%$ but a specificity of only $40 \%$. This means that if all men with a PSA $>4 \mathrm{ng} / \mathrm{ml}$ undergo prostate biopsies more than half will not be diagnosed with cancer, and indeed in the PSA range $4-10 \mathrm{ng} / \mathrm{ml}$ the detection rate is only about $25 \%$ (table 1$).^{45}$

Other clinical dilemmas have also become apparent as PSA testing becomes widespread. For example up to $45 \%$ of men with organ-confined prostate cancer have a PSA $<4 \mathrm{ng} / \mathrm{ml}^{6}{ }^{6}$ and therefore if these potentially curable men are to be diagnosed, methods of increasing the sensitivity of the test are vital. Some negative biopsies in men with PSA $>4 \mathrm{ng} / \mathrm{ml}$ are due to sampling error and the questions of whom to rebiopsy and when have also been the subject of intensive research (box 1).

A further problem has been that the rapid introduction of PSA testing into clinical practice has been accompanied by the development of numerous commercial assays; over 80 are available in Europe alone. Many have utilised the same reference ranges developed in initial studies without performing rigorous standardisation experiments, even though the variability between assays has been shown to be as high as $20 \%{ }^{7}$

In this review methods that have been used to improve the accuracy of PSA in diagnosing prostate cancer will be described (box 2). Firstly, so-called PSA derivatives (age specific PSA, PSA density, and PSA velocity) will be discussed. All utilise the standard method of PSA measurement, but combine this with another variable in order to improve sensitivity and specificity. The finding that the serum distribution of PSA (either free or bound to various proteins) is altered in prostate cancer has led to methods of refining its use based on these properties and these will be reviewed in the second section. Finally, new markers such as human kallikrein 2 (hK2) and prostate specific membrane antigen (PSMA), which are still in the early stages of clinical assessment, will be discussed.

Abbreviations: $A C T$, alpha-1-antichymotrypsin; $A 2 M$, alpha-2-macroglobulin; API, alpha-1-trypsin (or protein) inhibitor; $\mathrm{BPH}$, benign prostatic hyperplasia; fPSA, free PSA; hK2, human kallikrein 2; hK3, human kallikrein 3; PSA, prostate specific antigen; PSMA; prostate specific membrane antigen; tPSA, total PSA 


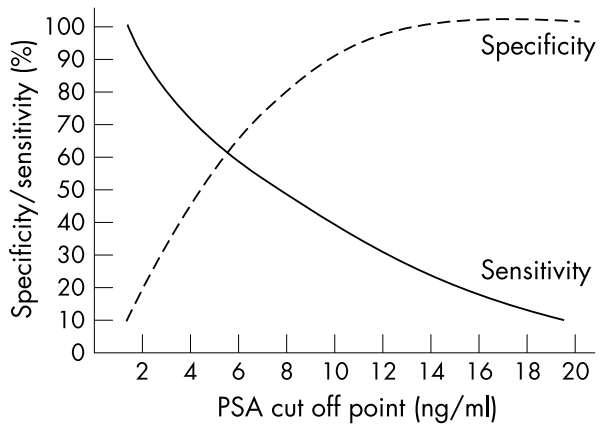

Figure 1 Sensitivity and specificity of the PSA test.

\section{Box 1: The aims of improving the utility of PSA in} prostate cancer diagnosis

- To reduce false positive results (increase specificity).

- To reduce false negative results (increase sensitivity).

- To indicate which men with negative prostate biopsies need rebiopsy and when.

Box 2: Proposed methods for improving diagnostic accuracy in prostate cancer

PSA derivatives

- Age specific PSA.

- PSA density.

- PSA velocity.

\section{Methods based on PSA binding}

- Free to total PSA ratio.

- Alpha-1-antichymotrypsin complexed PSA.

\section{Novel markers}

- Human kallikrein 2.

- Prostate specific membrane antigen.

\section{PSA DERIVATIVES}

\section{Age specific PSA}

It has been shown quite convincingly that PSA levels increase with increasing age. ${ }^{8}$ As well as the contribution from increasing prostate size this seems to be due to increased "leakiness" of the prostatic epithelium. This is thought to be due to subclinical inflammation or microscopic foci of cellular atypia. $^{9}$

Age specific PSA levels would therefore be expected to both increase the pickup of prostate cancer in younger men and reduce the number of biopsies performed in older men. Some guideline values are shown in table 2; these differ slightly between black and white men. ${ }^{10}$

Age specific PSA has been shown to increase detection of prostate cancer in younger men (50-59 years) by $15 \%$, although at a price of a $45 \%$ increase in the number of biopsies performed. ${ }^{11}$ This same study showed that in men over 70 years, because $44 \%$ fewer biopsies were performed, nearly half of all organ-confined cancers were missed. Partin et al found similar results but did observe that of the missed Tlc cancers (that is, impalpable and detected on random biopsy) in the older men, 95\% were of favourable histology and unlikely to be significant. ${ }^{12}$ Others have disputed this, ${ }^{13}$ and the use of age specific PSA values in men over 60 years remains controversial.

\section{PSA density}

The concept of PSA density is based on the assumption that, as non-neoplastic prostate tissue (especially BPH) does leak
Table 2 Age specific reference ranges for PSA ${ }^{10}$

\begin{tabular}{lll}
\hline & \multicolumn{2}{l}{ Normal PSA range $(\mathrm{ng} / \mathrm{ml})$} \\
\cline { 2 - 3 } Age (years) & Black men & White men \\
\hline $40-49$ & $0-2.0$ & $0-2.5$ \\
$50-59$ & $0-4.0$ & $0-3.5$ \\
$60-69$ & $0-4.5$ & $0-4.5$ \\
$70-79$ & $0-5.5$ & $0-6.5$ \\
\hline
\end{tabular}

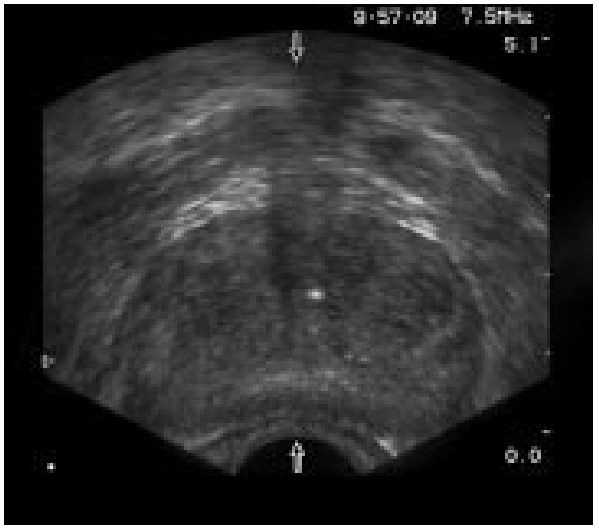

Figure 2 Transrectal ultrasound of the prostate.

some PSA into the circulation, for PSA to be useful in diagnosing prostate cancer, values should be corrected for total gland volume. Transrectal ultrasound is used to produce an estimate of gland volume (fig 2 ) and a threshold of 0.15 has been suggested as being discriminatory for cancer. ${ }^{14}$

Though clearly attractive in theory, initial reports showed that use of PSA density did not reduce the need for biopsy in the crucial group of patients with PSA 4-10 ng/ml. ${ }^{15}$ One group did suggest it might be of benefit in patients with PSA $>10 \mathrm{ng} / \mathrm{ml}^{16}{ }^{16}$ but this is a small group of patients with large prostates. Worryingly a recent report that has retrospectively looked at the accuracy of PSA density in a screened population has suggested that using a cut off of 0.15 may lead to over $30 \%$ of cancers being missed. ${ }^{17}$ Djavan et al suggested that measuring the volume of the transition zone of the prostate (the area most affected by BPH) might improve the accuracy of PSA density in men with PSA $4-10 \mathrm{ng} / \mathrm{ml}^{18}$ They demonstrated that if cut off values were adjusted so that $95 \%$ of cancers would be detected, the specificity of PSA transition zone was $47 \%$ compared with $26 \%$ for PSA density and only $6 \%$ for PSA alone. Despite this finding, the use of PSA density in this group of patients has not gained widespread acceptance and a major reason for this is the variability in the measurement of prostate volume with transrectal ultrasound.

An area where PSA density measurements may have more of a role is in deciding which men need a rebiopsy after an initial negative biopsy. ${ }^{19}$

\section{PSA velocity}

The rate of increase in serum PSA with time or PSA velocity is another method that has been proposed to improve performance of the assay. First data came from the Baltimore Longitudinal Ageing Study. ${ }^{20}$ This was a retrospective study on frozen serum specimens that suggested men with a PSA velocity of $>0.75 \mathrm{ng} / \mathrm{ml} /$ year had an increased chance of prostate cancer.

Problems with this method occur because of day to day variations in PSA, which can be up to $25 \%$ in the same individual. ${ }^{21}{ }^{22}$ Hence, in order to reliably detect a difference long periods (3-5 years) are required between measurements. 


\begin{tabular}{ll} 
Table 3 PSA distribution in serum & \\
\hline & Proportion (\%) \\
\hline Protein bound & $60-95$ \\
Alpha-1-antichymotrypsin (ACT)* & $60-90$ \\
Alpha-2-macroglobulin (A2M) & $10-20$ \\
Alpha-1-trypsin inhibitor (API)* & $1-5$ \\
Free* & $5-40$ \\
\hline *Detected in "total" PSA assays. & \\
\hline
\end{tabular}

It could not, therefore, be regarded as a safe method, as delaying biopsy in men with a PSA $>4 \mathrm{ng} / \mathrm{ml}$ means potential cures could be missed. The variability between different assays for PSA has been mentioned above and clearly if PSA velocity is to be of any practical use, follow up must be based on measurement in the same laboratory by the same method. Its main clinical utility is likely to be in older men $(>70)$ who are not candidates for radical surgery but in whom close observation is required in order to optimise the timing of hormonal therapy.

PSA velocity may be useful in monitoring men with a negative initial biopsy, and it has been suggested that a lower cut off value of $0.4 \mathrm{ng} / \mathrm{ml} /$ year be used as a threshold for rebiopsy in these patients. ${ }^{23}$ It may also be useful in those men with an initial PSA $<4 \mathrm{ng} / \mathrm{ml}$.

\section{METHODS BASED ON THE SERUM DISTRIBUTION OF PSA}

PSA that reaches the serum is either free or bound to plasma proteins (table 3 ). The most important binding proteins are alpha-1-antichymotrypsin (ACT), alpha-2-macroglobulin (A2M), and alpha-1-trypsin (or protein) inhibitor (API). Over 80 monoclonal antibodies have been described for the immunoassay of PSA and its molecular structure has been divided into six antigenic regions. When it is protein bound many of the epitopes on the PSA molecule are not available for antibody binding. Indeed when bound to A2M PSA is not detectable using standard methods (fig 3 ). Hence it has been possible to develop antibodies that recognise free PSA (fPSA) only.

\section{Free to total PSA ratio}

This was the first test to emerge based on the measurement of different PSA forms and relied on the development of antibodies that recognised only fPSA. It is important to bear in mind that while the conventional PSA tests purport to measure "total" PSA (tPSA) they do not measure that proportion bound to A2M. Hence the free to total (f/t) PSA ratio is effectively the ratio of fPSA to the sum of fPSA and that bound to ACT and API. ${ }^{24}$

$\mathrm{f} / \mathrm{t} \mathrm{PSA}=\mathrm{fPSA} /(\mathrm{fPSA}+\mathrm{ACT}$ PSA + API PSA $)$

In men with prostate cancer there seems to be a lower proportion of PPSA and this has been expressed as a decrease in the f/t PSA ratio. The reasons for this are not fully understood. Studies in 1991 demonstrated that there is a higher proportion of PSA bound to ACT in prostate cancer, ${ }^{25}{ }^{26}$ and it has been suggested that this is because of increased ACT production by prostate cancer cells. ${ }^{27}$ Another theory is that PSA from BPH is of a different isoform with multiple internal cleavages leading to an altered three dimensional structure that preferentially binds to A2M. ${ }^{28}$ As this is not detected by conventional tests for tPSA overall tPSA would be lower and hence the f/t PSA higher.

Initial clinical studies using the f/t PSA ratio were based on populations with a wide range of PSA values and while showing promising improvements in specificity were not clinically useful. Catalona et al in 1998 reported the results of a multicentre prospective trial designed to investigate the utility

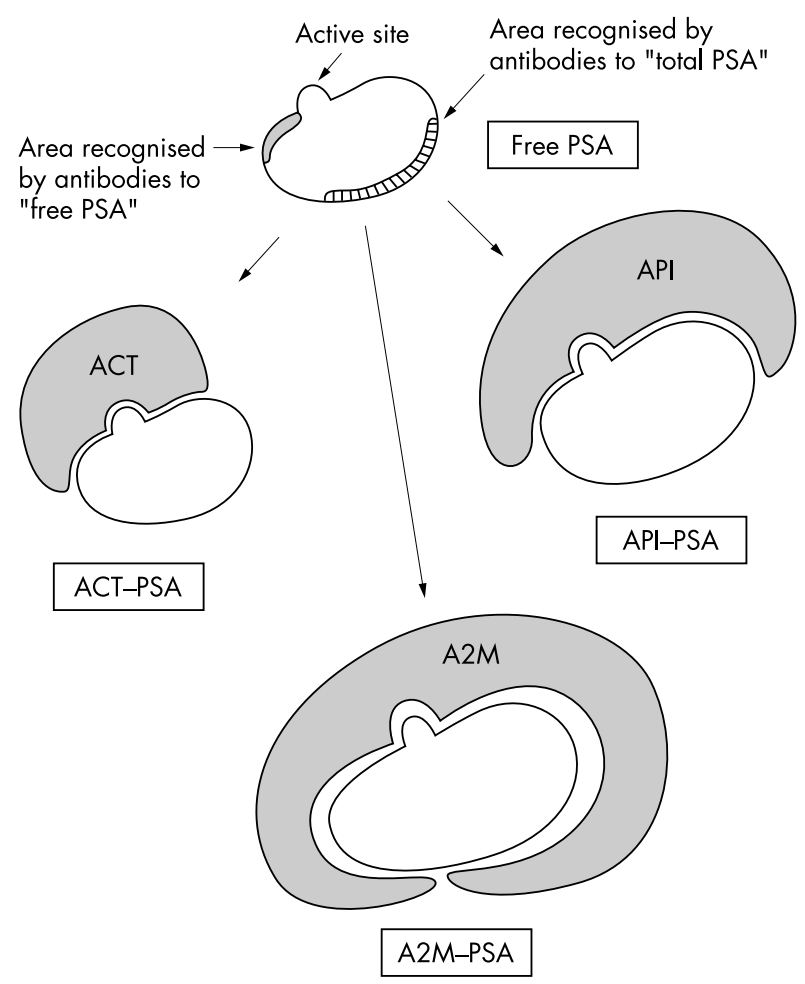

Figure 3 PSA protein binding.

of the $\mathrm{f} / \mathrm{t}$ PSA ratio in patients with a PSA in the range 4-10 $\mathrm{ng} / \mathrm{ml} .{ }^{29}$ They reported outcome data for two different cut off levels. Using a f/t PSA ratio of $>25 \%$ to determine biopsy, $95 \%$ of cancers were detected and $20 \%$ of unnecessary biopsies avoided. At a cut off of $22 \%$, sensitivity for detecting cancer dropped to $90 \%$ but unnecessary biopsies were now reduced by $29 \%$. These findings resulted in Food and Drug Administration approval of the f/t PSA for use in men with a PSA of 4-10 $\mathrm{ng} / \mathrm{ml}$.

When compared with other methods of deciding which men to rebiopsy after an initial negative biopsy f/t PSA has the best performance. ${ }^{30}$ In this study of 820 men with a PSA 4-10 $\mathrm{ng} / \mathrm{ml}$ and an initial benign biopsy, using a cut off of a f/t PSA ratio of $30 \%$ provides the best discrimination, detecting $90 \%$ of cancers and reducing unnecessary biopsies by $50 \%$.

Another scenario in which $\mathrm{f} / \mathrm{t}$ PSA ratio has shown promise is in men with PSA levels of less than $4 \mathrm{ng} / \mathrm{ml}$. At a f/t PSA cut off of $27 \%, 90 \%$ of cancers were detected and $18 \%$ of benign biopsies were avoided. ${ }^{31}$

Recently, an assay to measure A2M PSA has become available. The proportion of A2M PSA decreases in prostate cancer and combining this measurement with percentage free PSA enhances specificity of the test. ${ }^{32}$

\section{Alpha-1-antichymotrypsin complexed PSA}

As mentioned above, the reason for the decreased f/t PSA ratio in prostate cancer is thought, at least in part, to be due to an increase in binding to ACT. Until recently there have not been accurate assays for the measurement of ACT complexed PSA but these are now available. ${ }^{33}$ One theoretical advantage they have over the f/t PSA ratio is that only one variable is being measured. This would be expected to reduce the interassay variability seen with the use of the f/t PSA ratio, ${ }^{74}$ which it has been suggested might limit its widespread applicability. It is also likely to have benefits in terms of use of resources. A recent study has demonstrated that API complexed PSA actually falls in men with prostate cancer. ${ }^{35}$ As this contributes to the tPSA in conventional assays it might actually reduce the 
effectiveness of the f/t PSA ratio and is another reason that ACT complexed PSA might be expected to be an improvement.

Despite these theoretical advantages initial retrospective reports of the use of ACT complexed PSA showed no improvement in specificity compared to the use of the f/t PSA ratio. ${ }^{36}$ Indeed one study surprisingly reported it to be of decreased utility. ${ }^{37}$ Recently a prospective study has been reported and though the numbers were small (only 51 men with cancer) ACT complexed PSA did outperform $\mathrm{f} / \mathrm{t}$ PSA ratio with a specificity of $24.8 \%$ compared with $17.4 \%$, although this was not statistically significant.$^{38}$ Clearly larger prospective studies are required to fully evaluate the place of this new investigation.

\section{NOVEL MARKERS \\ Human kallikrein 2}

PSA belongs to the human kallikrein gene family, and another member hK2 is also found very specifically in the prostate. Its amino acid sequence shares $80 \%$ homology with that of PSA. The physiological role of hK2 is less clear, though it also has protease activity and may be involved in the activation of PSA. $^{39}$ Initial immunohistochemical studies demonstrated that hK2 expression in prostate cancer cells was increased compared with benign tissue, ${ }^{40}$ and it also correlated with higher Gleason grade.

The serum concentration of hK2 is approximately 100 times lower than that of PSA and so it has been important in the development of assays to ensure no cross reactivity. Also although hK2 does bind to serum proteins, unlike PSA $81 \%-96 \%$ is in the free form. ${ }^{41}$ Initial studies showed an increase in serum hK2 in patients with prostate cancer but measuring hK2 alone was no more discriminatory than the use of PSA. ${ }^{42}$ Subsequent work has looked into combining hK2 measurements with measurement of fPSA, and although retrospective these show some promise. Partin et al studied 937 men and found that using the hK2/fPSA ratio improved specificity over f/t PSA ratio. ${ }^{43}$ Another study used the ratio hK2 $\times$ tPSA/fPSA and also showed improved specificity over the $\mathrm{f} / \mathrm{t}$ PSA ratio alone. ${ }^{44}$

\section{Prostate specific membrane antigen}

PSMA is a transmembrane glycoprotein that is expressed on the surface of prostatic epithelial cells. It has been shown to be highly prostate specific, ${ }^{45}{ }^{46}$ and furthermore is expressed at higher levels in prostate cancer than in benign tissues. ${ }^{47}$

As PSMA is a membrane-bound antigen its detection in serum implies the presence of circulating prostate cells. Initial clinical studies were focused on the possibility of detecting cancer cells in the circulation in order to improve cancer staging by indicating those men in whom the cancer is unlikely to be organ-confined. Although they show promise, the sensitivity of the test varies from $39 \%$ to $91 \%$ in the studies reported suggesting further refinement is required. ${ }^{48}$

Serum levels of PSMA might be expected to be useful in the diagnosis of prostate cancer. Initial reports were based on the technique of western blotting, and while demonstrating increased expression in malignant compared with benign disease were not reliable for accurate quantitation and therefore not clinically applicable. ${ }^{49}$ A recent study has reported a novel immunoassay that allows quantitation. ${ }^{50}$ In men with a PSA of 4-10 ng/ml measuring PSMA was able to effectively differentiate those with BPH from those with cancer, although this was in a small number of patients (10 BPH and 17 cancer).

\section{CONCLUSIONS}

The diagnosis of prostate cancer was transformed 15 years ago with the introduction of the PSA test. Since that time a greater understanding of its behaviour and molecular biology has allowed refinements in its use such as those described in this article. While they show great promise none has yet become part of routine clinical practice. No doubt this reflects the relatively excellent sensitivity and specificity that PSA has as a tumour marker, making its use hard to improve on. Larger studies currently underway will confirm whether claims that complexed PSA has a higher specificity than the standard measurement will lead to it becoming the standard diagnostic test. It is clear that as consensus guidelines are developed for the management of prostate cancer it will be increasingly important to ensure that whatever diagnostic assay is used it is reproducible worldwide.

\section{QUESTIONS (ANSWERS AFTER REFERENCES) Q1. Prostate specific antigen is:}

(A) An enzyme secreted in the urine

(B) Present at increased levels in prostate cancer tissue

(C) Coded for by gene on chromosome 19

(D) Divided into eight antigenic regions

(E) In the same group of proteases as prostatic acid phosphatase

\section{Q2. Serum PSA levels:}

(A) Increase with increasing age

(B) Are raised in benign prostatic hyperplasia

(C) Fall in chronic prostatitis

(D) Are much lower than those in the semen

(E) Of $>4 \mathrm{ng} / \mathrm{ml}$ suggest a $75 \%$ chance of prostate cancer

\section{Q3. Which of the following statements are true:}

(A) Use of age related PSA ranges may reduce the biopsy rate in men over 70 by over $40 \%$

(B) PSA density is calculated by correcting PSA levels according to prostate volume estimated by digital rectal examination

(C) Use of PSA density does not reduce the need for biopsy in patients with PSA of $4-10 \mathrm{ng} / \mathrm{ml}$

(D) PSA density is made more useful by measuring the volume of the peripheral zone

(E) Men with a PSA velocity of over $0.75 \mathrm{ng} / \mathrm{ml} /$ year have an increased risk of prostate cancer

\section{Q4. With regard to serum measurement of PSA:}

(A) Day to day variations in the same individual are up to $25 \%$

(B) Over 80 antibodies exist for its estimation

(C) "Total" PSA does not include that fraction bound to alpha-1-chymotrypsin

(D) It exists most commonly in the "free" form

(E) Less than $5 \%$ is bound to alpha-1-trypsin inhibitor

\section{Q5. Human kallikrein 2}

(A) Shares $60 \%$ sequence homology with PSA

(B) Has serum levels $10 \times$ higher than those of PSA

(C) Exists in serum most commonly in the "free" form

(D) May be involved in PSA activation

(E) Serum levels have a greater sensitivity than PSA in the diagnosis of prostate cancer

\section{Authors' affiliations}

S Jain, A G Bhoiwani, J K Mellon, Division of Urology, University of Leicester, Leicester General Hospital, Leicester, UK

\section{REFERENCES}

1 Stamey TA, Yang N, Hay HR, et al. Prostate-specific antigen as a serum marker for adenocarcinoma of the prostate. N Engl J Med

1987;317:909-16. 
2 Diamandis EP, Yousef GM, Luo LY, et al. The new human kallikrein gene family: implications in carcinogenesis. Trends Endocrinol Metab 2000;11:54-60

3 Lilja H. A Kallikrein-like serine protease in prostatic fluid cleaves the predominant seminal vesicle protein. J Clin Invest 1985:76:1899-903.

4 Catalona WJ, Smith DS, Ratliff TL, et al. Measurement of prostate specific antigen in serum as a screening test for prostate cancer. N EnglJ Med 1991;324:1156-61.

5 Brawer MK, Chetner MP, Beatie J, et al. Screening for prostatic carcinoma with prostate specific antigen. J Urol 1992;147: 841-5.

6 Oesterling JE. Prostate specific antigen: a critical assesment of the most useful tumor marker for adenocarcinoma of the prostate. J Urol 1991; 145:907-23

7 Patel D, White PA, Ward AM. A comparison of six commercial assays for total and free prostate specific antigen (PSA): the predictive value of the ratio of free to total PSA. BJU Int 2000;85:686-9.

8 Oesterling JE, Jacobsen SJ, Chute CG, et al. Serum prostate-specific antigen in a community-based population of healthy men. Establishment of age-specific reference ranges. JAMA 1993;270:860-4.

9 Oesterling JE, Cooner WH, Jacobsen SJ, et al. Influence of patient age on the serum PSA concentration. An important clinical observation. Urol Clin North Am 1993;20:671-80.

10 Morgan TO, Jacobsen SJ, McCarthy WF, et al. Age-specific reference ranges for prostate-specific antigen in black men. N Engl J Med 1996:335:304-10.

11 Catalona WJ, Hudson MA, Scardino PT, et al. Selection of optimal prostate-specific antigen cutoffs for early detection of prostate-cancerreceiver operating characteristic curves. J Urol 1994;152:2037-42.

12 Partin AW, Criley SR, Subong EN, et al. Standard versus age-specific prostate specific antigen reference ranges among men with clinically localized prostate cancer: a pathological analysis. J Urol 1996; 155: 1336-9

13 Borer JG, Sherman J, Solomon MC, et al. Age specific prostate specific reference ranges: population specific. J Urol 1998;159:444-8.

14 Benson MC, Whang IS, Olsson CA, et al. The use of prostate specific antigen density to enhance the predictive value of intemediate levels of serum prostate specific antigen. J Urol 1992;147:817-21.

15 Brawer MK, Aramburu EA, Chen GL. The inability of PSA index to enhance the predictive value of PSA in the diagnosis of prostatic carcinoma. J Urol 1993:150:369-73.

16 Ohori M, Dunn JK, Scardino PT. Is prostate specific antigen density more useful than prostate specific antigen levels in the diagnosis of prostate cancer. Urology 1995;46:666-71.

17 Lujan M, Paez A, Llanes L, et al. Prostate specific antigen density. Is there a role for this parameter when screening for prostate cancer? Prostate Cancer and Prostatic Diseases 2001;4:146-9.

18 Diavan B, Zlotta AR, Byttebier G, et al. Prostate specific antigen density of the transition zone for early detection of prostate cancer. $J$ Urol 1998;160:411-8.

19 Catalona WJ, Beiser JA, Smith DS. Serum free prostate specific antigen and prostate specific antigen density measurements for predicting cancer in men with prior negative prostate biopsies. J Urol 1997;158:2162-7.

20 Carter HB, Pearson JD, Metter EJ, et al. Longitudinal evaluation of prostate specific antigen levels in men with and without prostate disease. JAMA 1992; 267:2215-20.

21 Nixon RG, Wener MH, Parson RE, et al. Biological variation of prostate specific antigen levels in serum: an evaluation of day-to-day physiological fluctuations in a well defined cohort of 24 patients. J Urol 1997:157:2183-90

22 Komatsu K, Wehner N, Prestigiacomo AF, et al. Physiologic (intraindividual) variation of serum prostate specific antigen in 814 men from a screening population. Urology 1996;47:343-6.

23 Smith DS, Catalona WJ. Rate of change in serum prostate specific antigen levels as a method for prostate cancer detection. J Urol 1994;152:1 163-7.

24 Zhang WM, Finne P, Leinonen J, et al. Measurement of the complex between prostate specific antigen and alpha 1 -protease inhibitor in serum. Clin Chem 1999;45:814-21.

25 Stenman U, Leinonen J, Alfthan $\mathrm{H}$, et al. A complex between prostate specific antigen and alpha 1-antichymotrypsin is the major form of prostate specific antigen in serum of patients with prostate cancer: assay of the complex improves clinical sensitivity for cancer. Cancer Res 1991:51:222-6.

26 Lilia H, Christensson A, Dahlen U, et al. Prostate specific antigen in serum occurs predominantly in complex with alpha- 1 antichymotrypsin. Clin Chem 1991;37:1618-25.

27 Biork T, Biartell A, Abrahamsson PA, et al. Alpha 1-antichymotrypsin production in PSA-producing cells is common in prostate cancer but rare in benign prostatic hyperplasia. Urology 1994;43:427-34.

28 Leinonen J, Zhang WM, Stenman U. Complex formation between PSA isoenzymes and protese inhibitors. J Urol 1996;155:1099-103.
29 Catalona WJ, Partin AW, Slawin KM, et al. Use of percentage of free prostate specific antigen to enhance differentiation of prostate cancer from benign prostatic disease. A prospective multicenter clinical trial. JAMA 1998:279:1542-7.

30 Diavan B, Zlotta A, Remzi M, et al. Optimal predictors of prostate cancer on repeat prostate biopsy: a prospective study of 1051 men. J Urol 2000;163: 1144-9.

31 Catalona WJ, Smith DS, Ornstein DK. Prostate cancer detection in men with serum PSA concentrations of 2.6 to $4.0 \mathrm{ng} / \mathrm{ml}$ and benign prostatic examination. JAMA 1997:277:1452-5.

32 Zhang WM, Finne P, Leinonen J, et al. Determination of prostate specific antigen complexed to alpha(2)-macroglobulin in serum increases the specificity of free to total PSA for prostate cancer. Urology 2000;56:267-72

33 Allard WJ, Zhou Z, Yeung KK. Novel immunoassay for the measurement of complexed prostate specific antigen in serum. Clin Chem 1998;44:1216-23.

34 Nixon RG, Gold $M H$, Blase $A B$, et al. Comparison of three investigative assays for the free form of prostate specific antigen. J Urol 1998; 160:420-5.

35 Finne $\mathbf{P}$, Zhang WM, Auvinen A, et al. Use for the complex between prostate specific antigen and alpha 1-protease inhibitor for screening prostate cancer. J Urol 2000;164:1956-60.

36 Brawer MK, Cheli CD, Neaman IE. Complexed prostate specific antigen provides significant enhancement of specificity compared with total prostate specific antigen for detecting prostate cancer. J Urol 2000; 163: 1476-80.

37 Stamey TA, Yemoto CE. Examination of the three molecular forms of prostate specific antigen for distinguishing negative from positive biopsy: Relationship to transition zone volume. J Urol 2000;163:119-26.

38 Mitchell ID, Croal BL, Dickie A, et al. Prospective study to evaluate role of complexed prostate specific antigen and free/total prostate specific antigen ratio for diagnosis of prostate cancer. J Urol 2001;165:1549 53.

39 Kumar A, Mikolajczyk SD, Goel AS, et al. Expression of pro form of prostate specific antigen by mammalian cells and its conversion to mature, active form by human kallikrein 2. Cancer Res 1997;57:3111-4

40 Darson MF, Pacelli A, Roche $P$, et al. Human glandular kallikrein 2 (hK2) expression in prostatic intraepithelial neoplasia and adenocarcinoma: a novel prostate cancer marker. Urology 1997;49:857-62.

41 Becker C, Piironen T, Kiviiemi J, et al. Sensitive and specific immunodetection of human glandular kallikrein 2 in serum. Clin Chem 2000;46: 198-206

42 Finlay JA, Evans CL, Day JR, et al. Development of monoclonal antibodies specific for human kallikrein 2 (hK2): development of dual antibody immunoassay for hK2 with negligible prostate specific antigen cross-reactivity. Urology 1998:51:804-9.

43 Partin AW, Catalona WJ, Finlay JA, et al. Use of human glandular kallikrein 2 for the detection of prostate cancer: preliminary analysis. Urology 1999;54:839-45

44 Becker C, Piironen T, Pettersson K, et al. Clinical value of human glandular kallikrein 2 and free and total prostate specific antigen in serum from a population of men with prostate specific antigen levels 3.0 $\mathrm{ng} / \mathrm{ml}$ or greater. Urology 2000;55:694-9.

45 Horoszewicz JS, Kawinski E, Murphy GP. Monoclonal antibodies to a new antigenic marker in epithelial prostatic cells and serum of prostate cancer patients. Anticancer Res 1987;7:927-36.

46 Israeli RS, Powell CT, Fair WR, et al. Molecular cloning of a complementary DNA encoding a prostate specific membrane antigen. Cancer Res 1993;3:227-30

47 Silver DA, Pellicer I, Fair WR, et al. Prostate specific membrane antigen expression in normal and malignant human tissues. Clin Cancer Res 1997:3:81-5.

48 Su SL, Boynton AL, Holmes EH, et al. Detection of extraprostatic prostate cells utilising reverse transcription polymerase chain reaction. Semin Surg Oncol 2000; 18:17-28.

49 Beckett ML Cazares LH, Vlahou A et al. Prostate specific membrane antigen (PMSA) levels in sera from healthy men or patients with benign prostatic hyperplasia or prostate cancer. Clin Cancer Res 1999;5:4034-40

50 Xiao Z, Bao-Ling A, Cazares LH, et al. Quantitation of serum prostate specific membrane antigen by a novel protein biochip immunoassay discriminates benign from malignant prostate disease. Cancer Res $2001 ; 61: 6029-33$

\section{ANSWERS}

Q1. C; Q2. A, B, D; Q3. A, C, E; Q4. A, B, E; Q5. C, D. 